Check for updates

Cite this: RSC Adv., 2020, 10, 7206

Received 8th November 2019

Accepted 31st January 2020

DOI: 10.1039/c9ra09271d

rsc.li/rsc-advances

\section{Synthesis of hyaluronic acid hydrogels by crosslinking the mixture of high-molecular-weight hyaluronic acid and low-molecular-weight hyaluronic acid with 1,4-butanediol diglycidyl ethert}

\begin{abstract}
Yu Xue, Hongyue Chen, Chao Xu, Dinghua Yu, (D) Huajin Xu and Yi Hu (D) *
High molecular weight hyaluronic acid (HMW-HA) and low molecular weight hyaluronic acid (LMW-HA) were mixed at different ratios and cross-linked with 1,4-butanediol diglycidyl ether (BDDE) to prepare five hyaluronic acid hydrogels A-E. Enzymolysis stability, swelling rate, crosslinking degree, rheological characteristics, BDDE residual rate, surface microstructure, and cytotoxicity of different hydrogels were investigated. The results showed that hydrogel B obtained by 10\% HA (w/v, HMW-HA and LMW-HA having a mass ratio of $4: 1)$ crosslinking with $1 \%$ BDDE $(v / v)$ had stronger in vitro anti-degradation ability, better mechanical properties and lower cytotoxicity than those prepared by mixing in different proportions. Hydrogel $\mathrm{B}$ has potential applications in regenerative medicine and tissue engineering.
\end{abstract}

\section{Introduction}

Hyaluronic acid (HA) is a natural glycosaminoglycan and an important component of extracellular matrix, which is biocompatible and biodegradable., ${ }^{1,2}$ The structure and biological characteristics of HA make it have special activity in the process of cell signaling, wound repair and somatic morphogenesis. ${ }^{3}$ Its degradation products have been described as angiogenic and beneficial for the formation of osteoclasts. ${ }^{4,5}$ It also acts as endogenous risk signals in cancer. ${ }^{6} \mathrm{HA}$ is widely used in biomedicine, and is a new kind of biological material with practical value. However, native HA networks are relatively weak in mechanical properties, they dissolve rapidly in water and are susceptible to hyaluronidase in the physiological environment. ${ }^{7}$ Physiologically, HA which accounts for one-third of total HA content is degraded and recombined daily in the body. ${ }^{8}$ At the same time, the native HA network will rapidly leave the injection site after injection, and the half-life after injection in the skin and joints is less than $24 \mathrm{~h} .{ }^{9}$ All these factors seriously limit the clinical application of HA. Therefore, it is of great significance to modify HA to obtain HA hydrogels with stronger stability and better mechanical properties. HA can be modified by crosslinking, ${ }^{10}$ grafting, ${ }^{11}$ linking with hydrophobic substances, ${ }^{12}$ by forming polyionic complexes with

School of Pharmaceutical Sciences, State Key Laboratory of Materials-Oriented Chemical Engineering, Nanjing Tech University, Nanjing 210009, China. E-mail: huyi@njtech.edu.cn

$\dagger$ Electronic supplementary information (ESI) available. See DOI: 10.1039/c9ra09271d polysaccharides, proteins or surfactants with opposite charges, ${ }^{13}$ or by permeating networks to produce self-assembled aggregates, nanoparticles and gels..$^{14}$ Selin et al. proved that the composite material composed of hyaluronic acid and natural polymer could be used as the reinforcement material of tissue engineering scaffolds, it was shown that such composite materials exhibited high thermal and mechanical stabilities. ${ }^{15}$ Angeloni et al. prepared a porous chitosan/HA composite and achieved a bone biomimetic scaffold by promoting bone tissue specific protein deposition from differentiated mesenchymal stem cells. ${ }^{16}$ Naumenko et al. proposed chitosan scaffolds that are typically doped with other supporting compounds which allow for mechanical strengthening, thus yielding composite biologically resistant scaffolds, to overcome the disadvantages of pure chitosan scaffolds such as mechanical fragility and low biological resistance. ${ }^{17}$

1,4-Butanediol diglycidyl ether (BDDE) can react with HA under strong base conditions to form stable covalent bonds and is a widely used crosslinking agent in the preparation of HA hydrogels. Under alkaline conditions, the epoxy groups of BDDE react preferentially with the hydroxyl group on the primary alcohol in $\mathrm{HA}^{18}$ Stellavato et al. ${ }^{19}$ found that when HMW-HA and LMW-HA were physically mixed in a $1: 1$ ratio and heat treated to form HA mixed complexes, their viscosity and in vitro resistance to enzymatic hydrolysis were improved. Herein, we hope that a new hydrogel which has strong in vitro anti-enzymatic hydrolysis, good mechanical properties and low cytotoxicity could be obtained by crosslinking HMW-HA and LMW-HA in different proportions with BDDE. Swelling rate, enzymatic hydrolysis stability, crosslinking rate, rheological 
characteristics, BDDE residual rate, surface microstructure, and cytotoxicity were investigated.

\section{Results and discussion}

\subsection{Enzyme hydrolysis stability}

Crosslinked HA hydrogels can be degraded by hyaluronidase from sheep testis, enzymatic hydrolysis condition of hydrogels A-E obtained by BDDE crosslinking with the mixture of HMWHA and LMW-HA in different ratio was shown in Fig. 1. Hydrogels D and E were completely degraded after $12 \mathrm{~h}$, hydrogels A and C were completely degraded after $24 \mathrm{~h}$, and hydrogels B were completely degraded after $72 \mathrm{~h}$. In addition to hydrogel B, the degradation rate of HA hydrogels increased with the decrease of HWM-HA content, and the degradation rate of HA hydrogel obtained by crosslinking with LMW-HA alone was the fastest. It shows that the mixture ratio of HMW-HA and LMW-HA has a significant effect on the anti-enzymatic hydrolysis performance of the hydrogels. In $10 \%(\mathrm{w} / \mathrm{v})$ system, the hydrogel B obtained when the mass ratio of HMW-HA and LMW-HA is $4: 1$ has the best anti-enzymatic hydrolysis performance. This phenomenon may be attributed to the reason that the intra HMW-HA chains hydrogen bonds and the intra HMWHA chains hydrogen bonds have been broken during the preparation process, leading to an entanglement of the H-HA and L-HA chains, which have preferential cooperative bonding between each other. ${ }^{19}$

\subsection{Swelling ratio}

Crosslinked hydrogels are highly water-swollen, they can swell in water under biological conditions, but do not dissolve. ${ }^{20}$ Several characteristics have been exhibited by this high swelling performance, like the low surface tension of surrounding biological liquid and the low mechanical stimulation of surrounding tissue, good diffusion capability including nutriment diffusing into the hydrogel and cell waste removed from the hydrogel. ${ }^{21}$ The ability of molecules to diffuse into or release

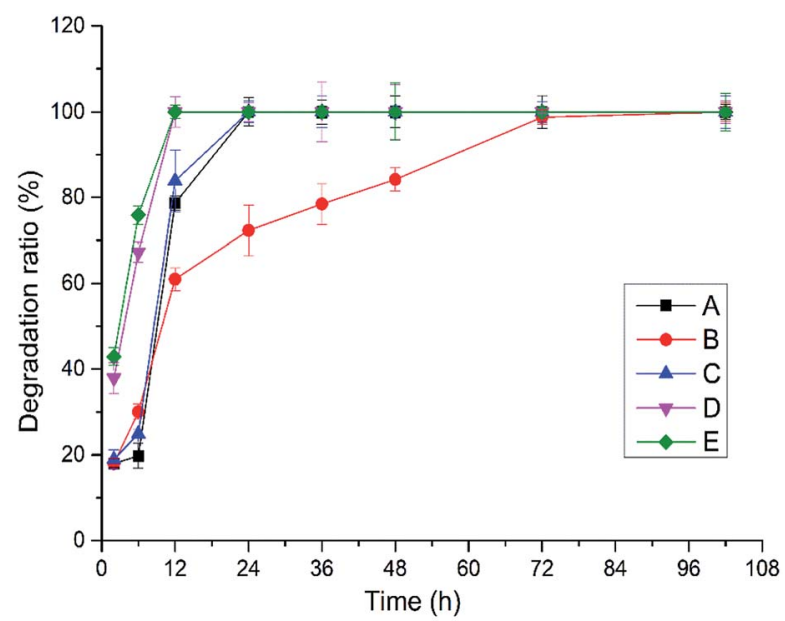

Fig. 1 Enzymatic hydrolysis of crosslinked hydrogels A-E prepared at different mixing ratio in vitro. from expanded hydrogels enables hydrogels to be used in a variety of biomedical applications including in skin enhancement, regenerative drugs, and carriers as bioactive substances. ${ }^{22}$ Therefore, basic measurement of the swelling capacity of hydrogels is critical.

Swelling ratio of crosslinked hydrogels A-E prepared at different mixing ratio in phosphate buffer saline (PBS, $\mathrm{pH}=7.2$, $0.02 \mathrm{~mol} \mathrm{l}^{-1}$ ) was shown in Fig. 2. The different water absorption capacity of hydrogels A-E at equilibrium state indicates that the different mixing ratio of HMW-HA and LMW-HA has certain influence on the expansion performance of hydrogels. The swelling ratio can reflect the degree of modification to a certain extent. The higher the crosslinking degree, the tighter the crosslinking network, and the smaller the swelling rate would be. ${ }^{23}$ As can be seen from Fig. 2, except for hydrogel B, the swelling ratio of HA hydrogels obtained by crosslinking increased with the increase of LMW-HA addition amount.

\subsection{Determination of crosslinking degree}

Crosslinking degree of crosslinked hydrogels A-E prepared at different mixing ratio was different and calculated by ${ }^{1} \mathrm{H}$ NMR analysis of the hydrolysate. The signal at $\delta=1.5 \mathrm{ppm}$ belongs to the $-\left(\mathrm{CH}_{2}\right) 2$ signal peak in the middle of BDDE structure. The signal peak $\delta=1.8 \mathrm{ppm}$ belongs to the $N$-acetyl group in HA. ${ }^{24}$ The chemical crosslinking degree of crosslinked HA (MoD) was calculated by eqn (1). The results were shown in Fig. 3, and the spectrum were shown in the ESI. $\dagger$

$$
\operatorname{MoD}(\%)=\frac{\left(I^{\delta H 1.5} / 4\right)}{\left(I^{\delta H 1.8} / 3\right)} \times 100
$$

The crosslinking degrees of HA hydrogels were ranked as E, B, D, C, and A in descending order. The result was basically consistent with swelling property in the previous section. In addition to hydrogel $\mathrm{B}$, the modification of hydrogels $\mathrm{A}-\mathrm{E}$ decreased with the addition of LMW-HA. The crosslinking degree of hydrogel B was significantly lower than that of hydrogel A prepared by HMW-HA alone. This phenomenon may

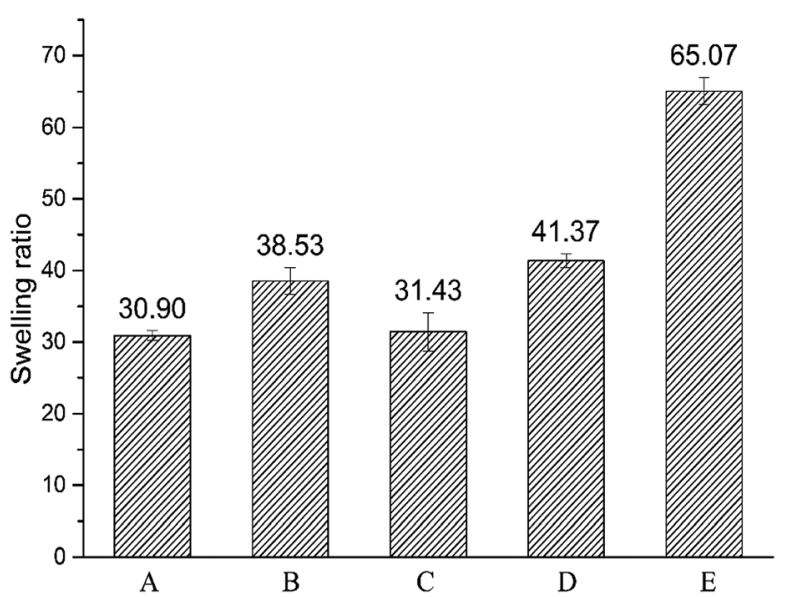

Fig. 2 Swelling ratios of crosslinked hydrogels $A-E$ prepared at different mixing ratio in phosphate buffer saline (PBS). 


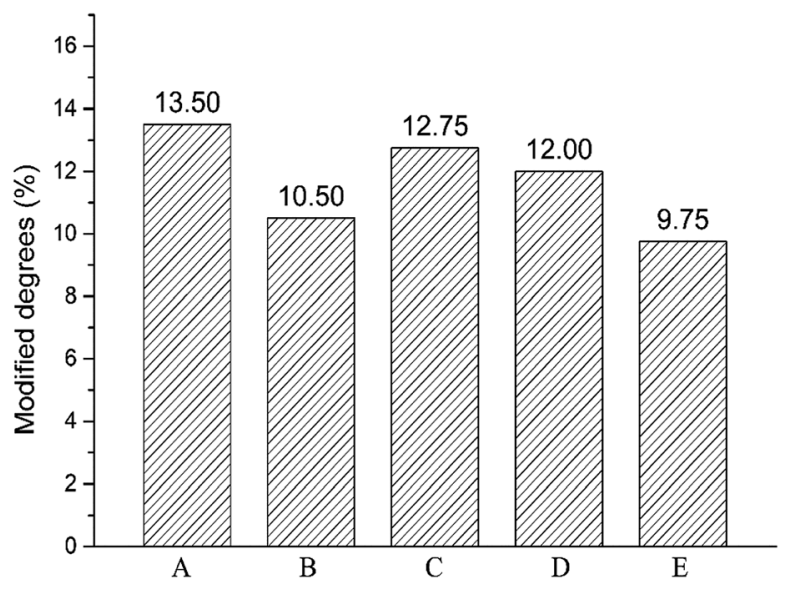

Fig. 3 Modified degrees of crosslinked hydrogels A-E prepared at different mixing ratio.

exist for the reason that the addition of LMW-HA will reduce the crosslinking efficiency and the overall mechanical properties of the hydrogel within a certain range. ${ }^{25}$ The high crosslinking degree of hydrogel A may lead to more BDDE residues, which could enhance the potential toxicity to cells.

\subsection{Rheological experiments}

The rheological properties of the hydrogel A-E were shown in Fig. 4, and some parameters were shown in Table 1. Storage
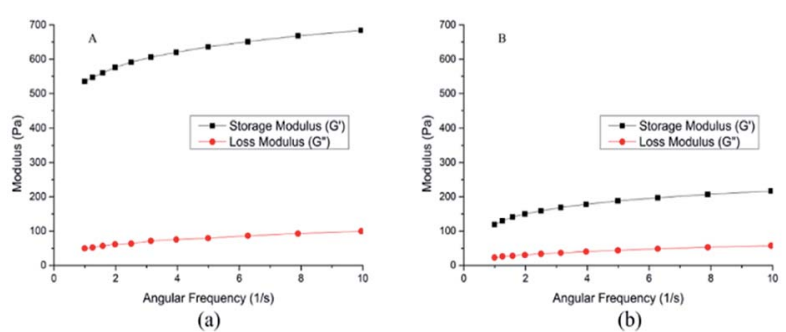

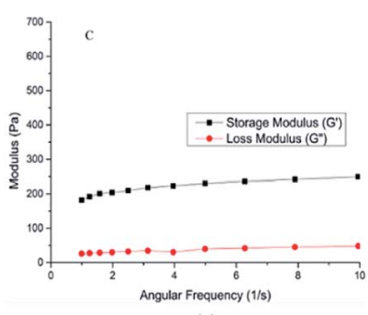

(c)

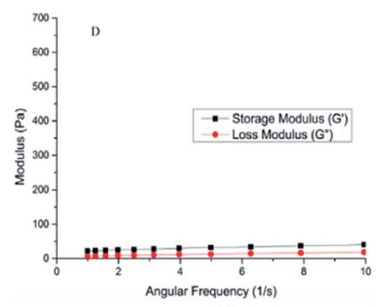

(d)

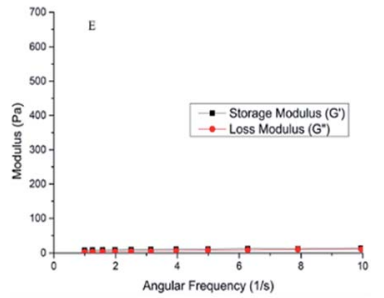

(e)

Fig. 4 Rheological properties of crosslinked hydrogels A-E prepared at different mixing ratio.
Table 1 Viscoelasticity of crosslinked hydrogels A-E prepared at different mixing ratio

\begin{tabular}{lrrrrr}
\hline & \multicolumn{2}{c}{$G^{\prime}(\mathrm{Pa})$} & & \multicolumn{2}{c}{$G^{\prime \prime}(\mathrm{Pa})$} \\
\cline { 2 - 3 } \cline { 6 - 6 } Entry & \multicolumn{1}{c}{$1 \mathrm{~Hz}$} & $10 \mathrm{~Hz}$ & & $1 \mathrm{~Hz}$ & $10 \mathrm{~Hz}$ \\
\hline A & 535.00 & 684.00 & & 49.70 & 99.60 \\
B & 119.00 & 217.00 & & 23.00 & 57.60 \\
C & 182.00 & 249.00 & & 26.00 & 48.20 \\
D & 22.00 & 40.60 & & 6.62 & 18.70 \\
E & 8.05 & 14.10 & 3.23 & 11.00 \\
\hline
\end{tabular}

moduli $\left(G^{\prime}\right)$ of the hydrogel A-E were higher than loss moduli $\left(G^{\prime \prime}\right)$, indicating that the hydrogel A-E had great viscoelasticity. ${ }^{26}$

The higher the storage modulus is, the greater the stiffness will be, the less deformation the material will be, and the stronger the brittleness will be. The lower the loss modulus is, the larger the deformation is, and the smaller the stiffness is. Therefore, hydrogel A was more brittle than hydrogel B, and hydrogel B was more flexible than hydrogel A, which meant hydrogel A with strong anti-enzymatic hydrolysis ability had better injection performance. ${ }^{23}$ As for $\mathrm{D}$ and E, the value of $G^{\prime}$ was close to the value $G^{\prime \prime}$, which represented that D ang E were semisolid with low mechanical strength.

\subsection{BDDE residues}

BDDE has certain biological toxicity and potential carcinogenicity. Therefore, BDDE residues of hydrogels were investigated. The results were shown in Table 2. The BDDE residual amount of the hydrogel $\mathrm{B}$ with the best enzymatic hydrolysis performance was lower than that of the hydrogel A.

\subsection{Scanning electron microscope (SEM)}

Although SEM cannot determine the presence of ether bonds in crosslinked hydrogels, it provides unique information about porosity and scaffold connectivity. Fig. 5 showed the SEM surface images of hydrogel A-E. SEM images showed the microstructure differences of hydrogels. Hydrogels A, B and D formed dense cross-linking networks with different pore structures. Hydrogels D had the largest pore structure, so it was easy to be hydrolyzed by enzymes. Hydrogel C formed large cracks in the freeze-drying process, possibly because the excessive amount of LMW-HA was added, which resulted in uneven distribution in the

Table 2 The residual amount of BDDE in crosslinked hydrogels A-E prepared at different mixing ratio in vitro

\begin{tabular}{lc}
\hline Entry & $\begin{array}{l}\text { The residual amount } \\
\text { of BDDE }\left(\mu \mathrm{g} \mathrm{g}^{-1}\right)\end{array}$ \\
\hline A & 10.42 \\
B & 8.18 \\
C & 3.23 \\
D & 3.01 \\
E & 4.99
\end{tabular}



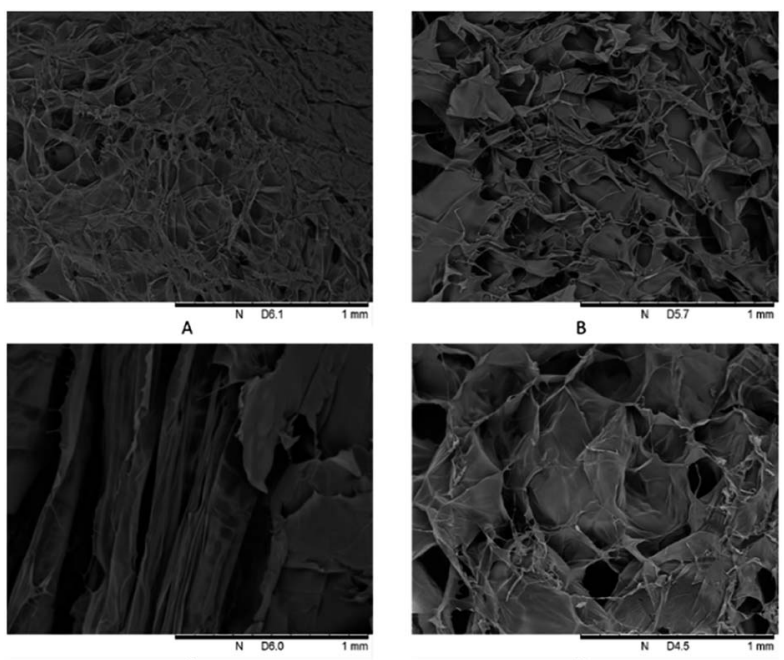

D

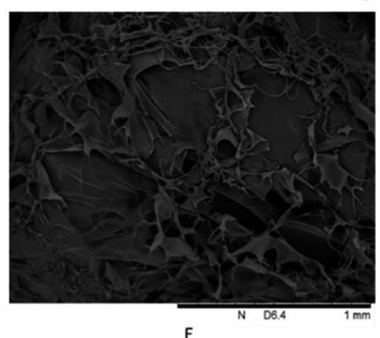

Fig. 5 SEM spectra of crosslinked hydrogels A-E prepared at different mixing ratio.

crosslinking process. The LMW-HA chain was relatively short and easy to fracture in the freeze-drying process. However, most layers were closely connected with each other, and hydrogel $\mathrm{C}$ also had relatively good anti-enzymatic hydrolysis ability. Hydrogel E was closer to the original HA image reported in the literature, ${ }^{27}$ in the process of freeze-drying, the extremely thin layered structure and loose pores were formed, and there was a certain degree of collapse. This may also be one of the reasons for its weak antidegradation ability.

\subsection{Cytotoxicity test}

From previous sections, a hydrogel with strong in vitro antidegradation ability, low BDDE residue and strong flexibility has been prepared without too much introduction of crosslinking agent (hydrogel B: 10\% HA (w/v, HMW-HA and LMW-HA mass ratio $4: 1), 1 \% \operatorname{BDDE}(\mathrm{v} / \mathrm{v}))$. To evaluate its safety as a potential biomedical material, MTT assay was used to determine its toxicity to mouse L929 fibroblasts. The cytotoxicity of hydrogel A prepared by HMW-HA alone under the same conditions was compared. Cytotoxicity was graded according to the relative cell proliferation rate, as shown in Table 3.

As shown in Table 4 and Fig. 8, the cytotoxicity of hydrogel A was grade 1 and grade 2 at most extraction concentrations, and at very low concentration of $0.1 \mathrm{mg} \mathrm{ml}^{-1}$, the cytotoxicity was grade 0 . The cytotoxicity of hydrogel B extracted at different concentrations was level 0. Compared with the blank control group, hydrogel B extracts at different concentrations promoted
Table 3 Relative growth rate and cytotoxicity grade

Relative cell

proliferation rate $(\%)$

Cytotoxical grade

$\geq 100$

$75-99$

$50-74$

25-49

$1-24$

$\leq 1$

0
1
2
3
4
5

the proliferation of L929 cells. In the positive control group, mouse L929 fibroblasts was cultured with phenol diluent, apoptosis was observed and the relative cell proliferation rate dropped to $26.680 \%$, indicating the cytotoxicity was level 3 . This indicated that hydrogel B promoted cell viability, which may be due to the small amount of LMW-HA in the leaching solution could bind to specific receptors and stimulate the proliferation of fibroblasts. ${ }^{28}$ It can be clearly seen from Table 5 that the cytotoxicity of hydrogel B was lower than that of hydrogel A.

As was shown in Fig. 6 and 7, blank control group L929 cells were flat spindle-shaped and angular, the morphology of cells in the positive control group (PC) was significantly changed, with a small amount of morphology remained, and the majority of cells were atrophic with fuzzy edges. As can be seen from Fig. 6, after incubation in hydrogel A extracts $\left(100 \mathrm{mg} \mathrm{ml}^{-1}, 20 \mathrm{mg}\right.$ $\mathrm{ml}^{-1}, 5 \mathrm{mg} \mathrm{ml}^{-1}, 1 \mathrm{mg} \mathrm{ml}^{-1}$ and $0.5 \mathrm{mg} \mathrm{ml}^{-1}$ ) for $24 \mathrm{~h}$, the morphology of L929 cells changed to some extent, resulting in cell atrophy and fuzzy or round edges, indicating that hydrogel A had relatively high biological toxicity. As can be seen from Fig. 7, after $24 \mathrm{~h}$ incubation in hydrogel B extract, no significant morphological changes were observed in L929 cells, which were fusiform with clear boundaries and complete morphology, similar to cells in the normal negative control group, preliminarily proving the biosafety of hydrogel $\mathrm{B}$. The results were consistent with the result of cytotoxicity grading.

\section{Experimental}

\subsection{Materials}

The high-molecular-weight HA sample (2000 kDa) and lowmolecular-weight sample $(200 \mathrm{kDa})$ were purchased from Bloomage Freda Biopharm Co. Ltd. (Shandong, China); 1,4butanediol diglycidyl ether (BDDE), (methyl sulfoxide)- $\mathrm{d}_{6}$ and $\mathrm{D}^{-}$ glucuronic acid were purchased from Meryer (Shanghai) Chemical Technology Co., Ltd. (Shanghai, China); $\mathrm{NaOH}, \mathrm{KOH}$, $\mathrm{NaH}_{2} \mathrm{PO}_{4} \cdot 2 \mathrm{H}_{2} \mathrm{O}, \mathrm{Na}_{2} \mathrm{HPO}_{4} \cdot 12 \mathrm{H}_{2} \mathrm{O}$, sodium tetraborate decahydrate and nicotinamide were purchased from Sinopharm Chemical Reagent Co. Ltd (Shanghai, China); hyaluronidase was purchased from Shanghai yuanye Bio-Technology Co. Ltd. (Shanghai, China); $\mathrm{H}_{2} \mathrm{SO}_{4}$ was purchased from Shanghai Lingfeng Chemical Reagent Co. Ltd (Shanghai, China); carbazole and formic acid were purchased from Shanghai Aladdin BioChem Technology Co. Ltd (Shanghai, China); acetophenone was purchased from Energy Chemical (Shanghai, China). All the chemicals were of analytical grade. 
Table 4 The OD values of each test group and the relative growth rate of L929 cells at $24 \mathrm{~h}$

\begin{tabular}{llll}
\hline & & & \multicolumn{2}{c}{$\begin{array}{l}\text { Relative growth } \\
\text { rate }(\%)\end{array}$} \\
\hline Eydrogel A & OD value $(\bar{x} \pm S)$ & 75.03 \\
& $100 \mathrm{mg} \mathrm{ml}^{-1}$ & $0.199 \pm 0.038$ & 69.182 \\
Cytotoxicity
\end{tabular}

\subsection{Methods}

3.2.1 Preparation of hyaluronic acid hydrogel crosslinked by BDDE. A total of $10 \%(\mathrm{w} / \mathrm{v})$ HA powders with different ratios of HMW-HA and LMW-HA solution were prepared by dissolving in $0.25 \mathrm{M} \mathrm{NaOH}$ with $1 \%(\mathrm{v} / \mathrm{v}) \mathrm{BDDE}$. After reaction at $40{ }^{\circ} \mathrm{C}$ for $6 \mathrm{~h}$, the reaction mixture was washed with deionized water 3 times to obtain the hydrogel product. The hydrogel was dialyzed against phosphate buffer (PBS) for $24 \mathrm{~h}$ then dialyzed with deionized water for $24 \mathrm{~h}$ to fully remove unreacted BDDE, lyophilized in a lyophilizer for $48 \mathrm{~h}$ to obtain white spongy samples. The composition list of crosslinked HA hydrogels are shown in Table 5.

3.2.2 Enzyme hydrolysis stability. $12 \mathrm{mg}$ lyophilized sample was swelled in PBS for $12 \mathrm{~h}$. The swelling hydrogel was mixed with $10 \mathrm{ml}$ hyaluronidase solution $\left(100 \mathrm{U} \mathrm{ml}^{-1}\right.$, dissolved in PBS), and then incubated at $42{ }^{\circ} \mathrm{C} .0 .5 \mathrm{ml}$ sample was taken at $2 \mathrm{~h}, 6 \mathrm{~h}, 12 \mathrm{~h}, 24 \mathrm{~h}, 36 \mathrm{~h}, 48 \mathrm{~h}, 72 \mathrm{~h}$ and $102 \mathrm{~h}$ from liquid supernatant. The enzyme in sample was deactivated in a boiling water bath for $10 \mathrm{~min}$. After cooling to room temperature, the sample was filtered through $0.22 \mu \mathrm{m}$ PTFE membrane, and the concentration of glucuronic acid in the filtrate was determined by Bitter-Muir method. ${ }^{29}$ The glucuronic acid concentration in supernatant was signed as $\mathrm{c} 1$ and the final concentration of glucuronic acid in the supernatant was signed as $c_{2}$. The degradation ratio was defined as the eqn (2).

Table 5 Composition list of raw material for HA hydrogels

\begin{tabular}{llllll}
\hline & A & B & C & D & E \\
\hline HMW-HA (g) & 0.5 & 0.4 & 0.375 & 0.333 & 0 \\
LMW-HA (g) & 0 & 0.1 & 0.125 & 0.167 & 0.5 \\
NaOH (ml) & 4.95 & 4.95 & 4.95 & 4.95 & 4.95 \\
BDDE $(\mu \mathrm{l})$ & 50 & 50 & 50 & 50 & 50
\end{tabular}

The degradation ratio $(\%)=c_{1} / c_{2} \times 100$

(2)

3.2.3 Swelling ratio. ${ }^{30}$ Lyophilized sample was immersed in phosphate buffer saline (PBS) and was taken out until equilibrium state. The excess water on the surface was removed by filter
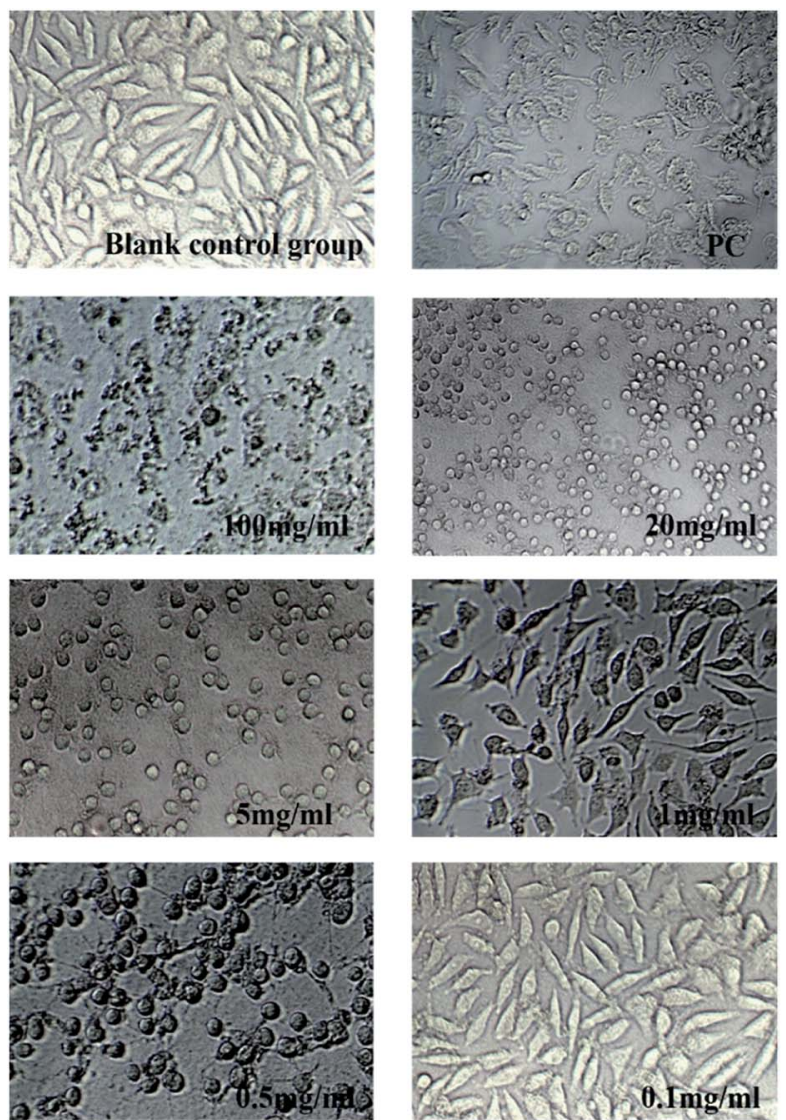

Fig. 6 Changes in cell morphology after incubated by Hydrogel $A$ extract for $24 \mathrm{~h}(\times 200)$. 

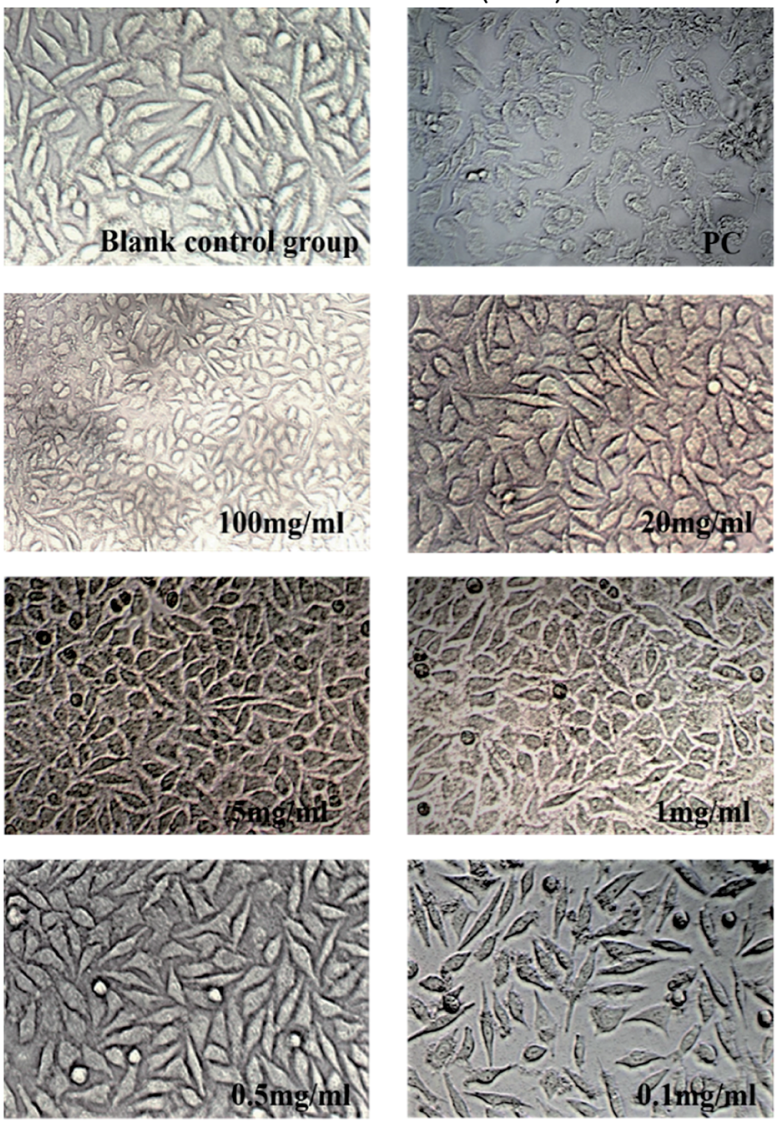

Fig. 7 Changes in cell morphology after incubated by Hydrogel B extract for $24 \mathrm{~h}(\times 200)$.

paper and sample was re-weighed. Swelling ratio was calculated by eqn (3).

$$
\text { Swelling ratio }\left(\mathrm{g} \mathrm{g}^{-1}\right)=W_{\mathrm{s}} / W_{\mathrm{d}}
$$

$W_{\mathrm{s}}$ is the weight of sample at equilibrium state and $W_{\mathrm{d}}$ is the weight of initial lyophilized sample.

3.2.4 Determination of crosslinking degree. Lyophilized sample was hydrolyzed by sulfuric acid solution $(0.5 \mathrm{M})$. The hydrolysate was then lyophilized, dissolved in $6 \mathrm{ml}$ DMSO- $\mathrm{d}_{6}$ and transferred into $5 \mathrm{~mm}$ NMR tubes. The NMR spectrum was measured on a Bruker Advance $300 \mathrm{MHz}$ instrument (Bruker, Germany) with DMSO- $\mathrm{d}_{6}$ as the solvent and TMS as internal standard.

3.2.5 Rheological experiments. Rheological experiments were performed using a Physica MCR302 oscillatory rheometer (Anton Paar, Germany) which equipped with a parallel plate geometry, $25 \mathrm{~mm}$ plate diameter, $1.0 \mathrm{~mm}$ gap, and a Peltier temperature control. The experiment temperature was $37{ }^{\circ} \mathrm{C}$. The strain value was $0.1 \%$ and oscillation frequency sweep tests were carried out over a frequency range from 1 to $10 \mathrm{~Hz}$.

3.2.6 BDDE residues. ${ }^{31}$ BDDE residues can be tested by detecting fluorescence intensity of the substance produced by BDDE and nicotinamide, which has strong fluorescent where excitation wavelength and emission wavelength were located at

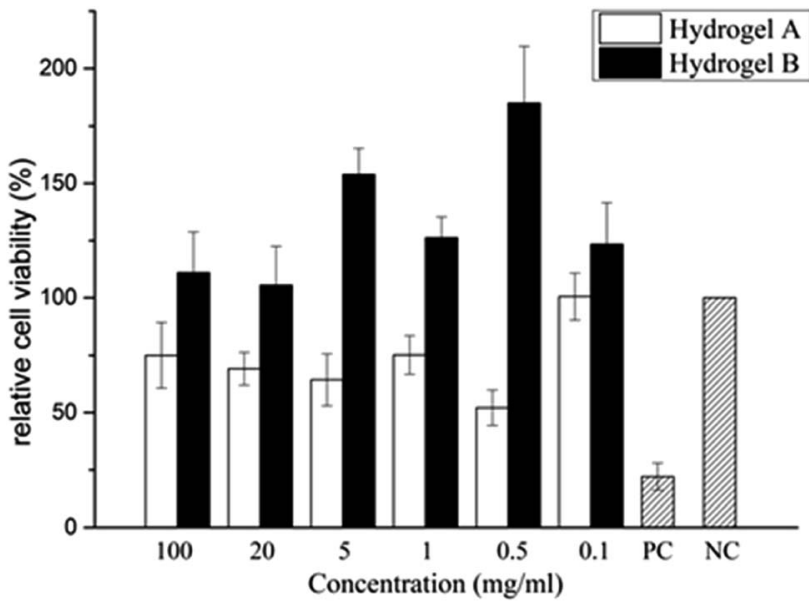

Fig. 8 Changes of relative cell viability after incubated by HA hydrogel extract for $24 \mathrm{~h}$.

$370 \mathrm{~nm}$ and $430 \mathrm{~nm} .20 \mu \mathrm{l} 8.0,4.0,2.0,1.0,0.5,0.25 \mu \mathrm{g} \mathrm{ml} \mathrm{m}^{-1}$ BDDE solution and $10 \mu \mathrm{l} 125 \mathrm{mM} \mathrm{l}^{-1}$ nicotinamide solution were mixed respectively in tubes and incubated $2 \mathrm{~h}$ at $37^{\circ} \mathrm{C} .100$ $\mu \mathrm{l} 15 \%$ acetophenone solution ( $\mathrm{w} / \mathrm{w}$, dissolved in ethanol) and $100 \mu \mathrm{l} 1 \mathrm{~mol} \mathrm{l}^{-1}$ potassium hydroxide solution were added into tubes, set on ice for $10 \mathrm{~min}$. Then $0.5 \mathrm{ml}$ formic acid was added into tubes and incubated for $5 \mathrm{~min}$ at $60{ }^{\circ} \mathrm{C}$. The fluorescence values were determined by Multifunctional microplate reader SpectraMax M5 (Thermo Fisher Science, USA) where excitation wavelength and emission wavelength were located at $370 \mathrm{~nm}$ and $430 \mathrm{~nm}$. BDDE concentration and fluorescence were used as the abscissa and the ordinate to make the standard curve. $1 \mathrm{~g}$ swelling HA hydrogels were hydrolyzed by $1 \mathrm{ml}$ hyaluronidase solution (100 $\mathrm{U} \mathrm{ml}^{-1}$ ). The BDDE content of enzymatic hydrolysate was determined by the method above.

3.2.7 Scanning electron microscope (SEM). Lyophilized sample was first deposited on aluminum stubs and then coated with gold using an ion sputter and then visualized by scanning electron microscope (SEM) from Hitachi Tabletop Microscope (TM3000, Tokyo, Japan).

3.2.8 Cytotoxicity test. In order to evaluate safety of hydrogels as a potential biomedical material, MTT method has been used to determine the cytotoxicity of hydrogels and the effect on cell morphology. ${ }^{32}$ L929 mouse fibroblasts were purchased from CAS typical culture collections Committee cell library (Shanghai, China). Cells were cultured in minimum essential medium (MEM) with 10\% (w/w) FBS and 1\% (w/w) penicillin/streptomycin at $37{ }^{\circ} \mathrm{C}$ in a humidified atmosphere of $5 \% \mathrm{CO}_{2}$. Extract liquid was prepared by extracting the hydrogel sample at $37{ }^{\circ} \mathrm{C}$ with culture medium for $24 \mathrm{~h}$. L929 mouse fibroblasts $\left(2.5 \times 10^{4}\right.$ cells per $\left.\mathrm{cm}^{2}\right)$ were cultured in 96well microplates for $24 \mathrm{~h}$. Then medium was discarded, cells were treated with $100 \mathrm{mg} \mathrm{ml}^{-1}, 20 \mathrm{mg} \mathrm{ml}^{-1}, 5 \mathrm{mg} \mathrm{ml}^{-1}, 1 \mathrm{mg}$ $\mathrm{ml}^{-1}, 0.5 \mathrm{mg} \mathrm{ml}{ }^{-1}, 0.1 \mathrm{mg} \mathrm{ml} \mathrm{m}^{-1}$ extract liquid for $24 \mathrm{~h}$, respectively. Equal volume of culture medium was used as blank control and equal volume of phenol diluent $(1 \%, v / v)$ as positive control. Cell morphology was recorded by Leica 
DMI3000B manual inverted microscope (Leica Microsystems, Germany). Subsequently, cells were stained with MTT at final concentration of $0.5 \mathrm{mg} \mathrm{ml}^{-1}$ in PBS ( $\left.\mathrm{pH} 7.4\right)$ for $4 \mathrm{~h}$ in dark and then the medium was discarded. The formazan crystals presented in cells were dissolved by $100 \mu$ l of DMSO. The absorbance was read at $490 \mathrm{~nm}$ on a Multiskan MK3 microplate reader (Thermo Fisher Science, USA). The relative growth rate (RGR) was calculated by eqn (4).

$$
\mathrm{RGR}=\frac{\text { absorbance values of treatment }}{\text { absorbance values of blank control }}
$$

\section{Conclusions}

HA hydrogels were prepared by crosslinking the mixture of HMW-HA and LMW-HA at different ratio with BDDE under alkaline conditions, and compared with that prepared by crosslinking HMW-HA or LMW-HA with BDDE alone. In vitro anti-enzymatic hydrolysis, swelling and rheological properties were investigated. The crosslinking degree and residue of crosslinking agent BDDE in hydrogels were determined, and the surface morphology was investigated by SEM. A novel crosslinked HA hydrogel B with strong in vitro anti-degradation ability, low BDDE residue and strong mechanic strength and flexibility could be obtained by $10 \% \mathrm{HA}$ (w/v, HMW-HA and LMW-HA have a mass ratio of $4: 1$ ) crosslinking with 1\% BDDE (v/v). The cytotoxicity of hydrogel B was determined by MTT assay, the effect of the extracts on cell morphology was investigated, and the hydrogel A obtained by HMW-HA crosslinking with BDDE under the same conditions was compared. The results showed that the cytotoxicity level of hydrogel B was 0 , which promoted the proliferation of L929 cells to a certain extent, and had no significant effect on the morphology of L929 cells. Hydrogel B has better biological safety than hydrogel A, and can be used as potential biomaterials in biomedical and other fields.

\section{Conflicts of interest}

There are no conflicts to declare.

\section{Acknowledgements}

This work was supported by the National Natural Science Foundation of China (No. 21676143), Self-Owned Research Project from Key Laboratory of Materials-Oriented Chemical Engineering (No. ZK201603), Jiangsu Synergetic Innovation Center for Advanced Bio-Manufacture, and Qing Lan Project of Jiangsu Province.

\section{Notes and references}

1 J. L. Drury and D. J. Mooney, Biomaterials, 2003, 24(24), 4337. 2 A. Olejnik, J. Goscianska, A. Zielinska and I. Nowak, Int. J. Cosmet. Sci., 2015, 37(4), 401.
3 J. Necas, L. Bartosikova, P. Brauner and J. Kolar, Vet. Med., 2008, 53(8), 397.

4 M. Slevin, J. Krupinski, J. Gaffney, S. Matou, D. West, H. Delisser, R. C. Savani and S. Kumar, Matrix Biol., 2007, 26(1), 58.

5 W. Ariyoshi, T. Takahashi, T. Kanno, H. Ichimiya, H. Takano, T. Koseki and T. Nishihara, J. Biol. Chem., 2005, 280(19), 18967.

6 J. Iijima, K. Konno and N. Itano, Cancers, 2011, 3(3), 3189.

7 R. Stern, A. A. Asari and K. N. Sugahara, Eur. J. Cell Biol., 2006, 85(8), 699.

8 C. W. Chung, J. Y. Kang, I. S. Yoon, H. D. Hwang, P. Balakrishnan, H. J. Cho, K. D. Chung, D. H. Kang and D. D. Kim, Colloids Surf., B, 2011, 88(2), 711.

9 T. J. Brown, U. B. G. Laurent and J. R. E. Fraser, Exp. Physiol., 1991, 76(1), 125.

10 C. E. Schante, G. Zuber, C. Herlin and T. F. Vandamme, Carbohydr. Polym., 2011, 85(3), 469.

11 M. H. Abu Elella, R. R. Mohamed and M. W. Sabaa, Carbohydr. Polym., 2018, 189, 107.

12 X. D. Wang, X. Q. Gu, H. M. Wang, Y. J. Sun, H. Y. Wu and S. R. Mao, Eur. J. Pharm. Sci., 2017, 96, 255.

13 J. X. Chen, M. Wang, H. H. Tian and J. H. Chen, Colloids Surf., B, 2015, 134, 81.

14 P. Matricardi, C. Di Meo, T. Coviello, W. E. Hennink and F. Alhaique, Adv. Drug Delivery Rev., 2013, 65(9), 1172.

15 S. S. Suner, S. Demirci, B. Yetiskin, R. Fakhrullin, E. Naumenko and O. Oguz, Int. J. Biol. Macromol., 2019, $627,635$.

16 V. Angeloni, N. Contessi, C. D. Marco, S. Bertoldi, M. C. Tanzi and M. G. Daidone, Acta Biomater., 2017, 306, 316.

17 E. A. Naumenko, I. D. Guryanov, R. Yendluri, Y. M. Lvova and R. F. Fakhrullin, Nanoscale, 2016, 8, 7257.

18 K. De Boulle, R. Glogau, T. Kono, M. Nathan, A. Tezel, J. X. Roca-Martinez, S. Paliwal and D. Stroumpoulis, Dermatol. Surg., 2013, 39(12), 1758.

19 A. Stellavato, L. Corsuto, A. D'Agostino, A. La Gatta, P. Diana, P. Bernini, M. De Rosa and C. Schiraldi, PloS One, 2016, 11(10), e0163510.

20 J. M. Zhu and R. E. Marchant, Expet Rev. Med. Dev., 2011, $8(5), 607$.

21 O. Jeon, S. J. Song, K. J. Lee, M. H. Park, S. H. Lee, S. K. Hahn, S. Kim and B. S. Kim, Carbohydr. Polym., 2007, 70(3), 251.

22 C. S. Satish, K. P. Satish and H. G. Shivakumar, Indian J. Pharm. Sci., 2006, 68(2), 133.

23 A. Borzacchiello, L. Russo, B. M. Malle, K. SchwachAbdellaoui and L. Ambrosio, BioMed Res. Int., 2015, 2015, 871218.

24 F. J. Wende, S. Gohil, L. I. Nord, A. H. Kenne and C. Sandstrom, Carbohydr. Polym., 2017, 157, 1525.

25 S. Ibrahim, Q. K. Kang and A. Ramamurthi, J. Biomed. Mater. Res., Part A, 2010, 94(2), 355.

26 J. Shin, J. S. Lee, C. Lee, H. J. Park, K. Yang, Y. Jin, J. H. Ryu, K. S. Hong, S.-H. Moon, H. M. Chung, H. S. Yang, S. H. Um, J. W. Oh, D. I. Kim, H. Lee and S. W. Cho, Adv. Funct. Mater., 2015, 25(25), 3814. 
27 M. Al-Sibani, A. Al-Harrasi and R. H. Neubert, Eur. J. Pharm. Sci., 2016, 91, 131.

28 A. Sparavigna and B. Tenconi, Clin., Cosmet. Invest. Dermatol., 2016, 9, 297.

29 T. Bitter and H. M. Muir, Anal. Biochem., 1962, 4(4), 330.

30 A. G. Ibrahim, F. A. Hai, H. A. Wahab and H. Mahmoud, Am. J. Appl. Chem., 2016, 4(6), 221-234.
31 H. Feng, G. Zhang and Y. Xu, Chin. J. Pharm. Anal., 2012, 32(4), 654.

32 D. T. Wu, L. Z. Meng, L. Y. Wang, G. P. Lv, K. L. Cheong, D. J. Hu, J. Guan, J. Zhao and S. P. Li, Carbohydr. Polym., 2014, 110, 405. 